(n)

OPEN ACCES $@$ @i)

\title{
Microbial communities of wild-captured Kemp's ridley (Lepidochelys kempii) and green sea turtles (Chelonia mydas)
}

\author{
Kerry L. McNally ${ }^{1,2, *}$, Cody R. Mott ${ }^{3}$, Jeffrey R. Guertin ${ }^{3}$, Jennifer L. Bowen ${ }^{4}$ \\ ${ }^{1}$ Animal Health Department, New England Aquarium, Boston, Massachusetts 02110, USA \\ ${ }^{2}$ University of Massachusetts, Boston, Massachusetts 20125, USA \\ ${ }^{3}$ Inwater Research Group, Inc., Jensen Beach, Florida 34957, USA \\ ${ }^{4}$ Department of Marine and Environmental Sciences, Marine Science Center, Northeastern University, Nahant, \\ Massachusetts 01908, USA
}

\begin{abstract}
Conservation efforts for endangered sea turtle species, such as Kemp's ridley turtles Lepidochelys kempii and green turtles Chelonia mydas, may benefit from information on the microbial communities that contribute to host health. Previous studies examining host-associated microbiomes of these species have been limited in geographic region, life stage, and/or health. Here, we characterized the microbiome of the oral cavity and cloaca from wild-captured Kemp's ridley and green turtles off the west coast of Florida, USA, by using Illumina sequencing to analyze the 16S rRNA gene. Microbial communities were distinct between body sites as well as between turtle species, suggesting that the turtle species is more important than the local environment in determining the microbiome of sea turtles. We identified the core microbiome for each species at each body site and determined that there were very few bacteria shared among the oral samples of both species, and no taxa co-occurred in the cloaca samples among both species. The core microbiome of the green turtle cloaca was primarily from the order Clostridiales, which plays an important role in digestion for other herbivorous species. Due to high prevalence of fibropapillomatosis in the green turtles $(90 \%)$, we also investigated the correlation between the microbiome and the severity of fibropapillomatosis, and we identified changes in beta diversity associated with the total number of tumors. This study provides the first glimpse of the microbiome in 2 sympatric species of sea turtle and sheds an important species-specific light on the microbiome of these endangered species.
\end{abstract}

KEY WORDS: Sea turtle $\cdot$ Microbiota $\cdot$ Fibropapillomatosis $\cdot$ Lepidochelys kempii $\cdot$ Chelonia mydas

\section{INTRODUCTION}

Microbes are considered a fundamental part of the life history of animals (McFall-Ngai et al. 2013, Colston \& Jackson 2016), including sea turtles. Sea turtles have complex life histories involving a diversity of habitats, including nesting on beaches, initial de-

${ }^{*}$ Corresponding author: kmcnally@neaq.org velopment in the open ocean, and foraging in coastal waters (Bolten 2003). These complex life histories are each susceptible to unique environmental pressures, including anthropogenic disturbances such as pollution and habitat destruction (Seminoff 2004, Wibbels \& Bevan 2019). Globally, the International Union for the Conservation of Nature (IUCN) lists the green

(C) The authors 2021. Open Access under Creative Commons by Attribution Licence. Use, distribution and reproduction are unrestricted. Authors and original publication must be credited. 
turtle Chelonia mydas as Endangered (Seminoff 2004) and the Kemp's ridley turtle Lepidochelys kempii as Critically Endangered (Wibbels \& Bevan 2019). Understanding the microbiome of these imperiled species would provide useful information concerning their biology and health monitoring.

Microbial communities play a role in host development and function, including nutrition, metabolism, immune response, behavior, and sociality (Ley et al. 2008b, Amato 2013, McFall-Ngai et al. 2013, Bordenstein \& Theis 2015, Colston \& Jackson 2016). Thus far, studies of sea turtle microbial communities have primarily focused on the gut of loggerhead or green turtles (Abdelrhman et al. 2016, Ahasan et al. 2017, 2018, Price et al. 2017, Campos et al. 2018, Biagi et al. 2019, Bloodgood et al. 2020, McDermid et al. 2020, Scheelings et al. 2020c), although studies on other species are becoming more common (Samuelson et al. 2020, Scheelings et al. 2020a,b,c). Variations in the cloacal microbiome of green turtles were evident between different habitats (pelagic vs. neritic), which suggests that environmental and dietary factors contribute to microbial community composition in that turtle species (Price et al. 2017, Campos et al. 2018). The fecal microbiota of loggerhead turtles studied during rehabilitation highlighted turtle resilience to a captive environment and showed distinct differences compared to the green turtle (Biagi et al. 2019). A more recent study of the gastrointestinal microbiota encompassed all species of sea turtles, but it is difficult to compare across species when all were adult nesting females from different locations in the world (Scheelings et al. 2020a). Microbial communities of nesting flatback turtles Natator depressus and loggerhead turtles differ based on populations (Scheelings et al. 2020b,c), highlighting that sampling turtles from different geographic regions is still important, but creates limitations in understanding species differences. Thus, additional studies on wild turtles and on a variety of turtle species, including the critically endangered Kemp's ridley turtle, are needed to assess whether turtles have a core microbial community of taxa that are found in the majority of individuals, or whether the microbiome varies at different life stages, habitats, or in different environments.

Determining the nature of the core microbiota of an organism is important for understanding the healthy and stable microbiome of hosts, including sea turtles. A core microbiome is defined as the microbes that are common or consistent across microbial communities of similar habitats (Turnbaugh et al. 2007, Shade \& Handelsman 2012). Core microbiome analysis is useful as a first step in identifying the healthy microbes of a host, thus allowing for monitoring organism health or predicting potential perturbations and/or the effects of dysbiosis (Shade \& Handelsman 2012, Apprill et al. 2014). For example, in healthy humpback whale respiratory vapor and skin, a core microbiome has been identified for each body site, which is important for assessing atypical microbes or the absence of members of the typical core communities (Apprill et al. 2014, 2017). Characterizing an animal's specific core microbial community can also be used to develop a screening tool that could identify host health, immunity, and disease. Establishing the core microbiome of endangered species of sea turtles could lead to improvements in conservation and rehabilitation management through monitoring this indicator of overall host health. Diets vary drastically between sea turtle species, each highly adapted to a specific dietary niche, making it likely that each species has a specific core microbiome. For example, green and Kemp's ridley turtles both inhabit the shallow coastal areas along the northwest coast of Florida, USA, where juvenile green turtles transition from being omnivores to herbivores in shallow seagrass beds, and juvenile and subadult Kemp's ridley turtles, which are carnivores, primarily feed on crustaceans (Bjorndal 1997, Arthur et al. 2008, Hoopes et al. 2017). The differences between the species' microbiomes remain to be explored.

Further investigation into diseases of sea turtles and the microbiome also remains important. Like other marine vertebrates, sea turtles are considered sentinels of ecosystem health due to their wide distribution, long lifespans, and occurrence in multiple ecosystems. Fibropapillomatosis (FP) is one prominent indicator of sea turtle population health, with its distribution and prevalence increasing over the last several decades (Jones et al. 2016). This infectious disease is found in all species of sea turtle, although it has reached epizootic proportions in green turtles (Aguirre \& Lutz 2004, Page-Karjian et al. 2019). FP is characterized by proliferative fibroepithelial lesions, and the likely etiological agent is the herpesvirus, chelonid herpesvirus 5 (ChHV5) (Wyneken et al. 2006, Jones et al. 2016). There are multiple factors that influence the expression of the virus by causing disruptions in the immune system, including environmental co-factors such as water quality, temperature, pollutants or toxins, and algal blooms (Wyneken et al. 2006, Jones et al. 2016, Page-Karjian \& Herbst 2017). The interactions between FP and the turtle microbiome remain unclear; however, documenting these interactions is a critical step in understanding the consequences of FP for host health. 
In this study, we investigated the microbiome of 2 endangered sea turtle species from the same environment. Our objective was to characterize the oral and cloacal microbiome of wild Kemp's ridley and green turtles from the same habitat. Sampling from the same habitat allowed us to control for local environmental variation that may influence the microbiome. We hypothesized that the turtle species would have distinct microbial communities from each other but that there would be a core microbiome that exists for each body site. Additionally, we investigated microbial community differences based on severity of FP. We hypothesized that there would be a relationship between the microbial community composition and severity of disease due to the effects of sequelae from FP and likely links between immune system function and the microbiome.

\section{MATERIALS AND METHODS}

\subsection{Sample collection}

Animal sampling was approved by the New England Aquarium (NEAq) Institutional Animal Care and Use Committee (Protocol \#2017-07), and we collected samples under NMFS permit \#16598-03 and Florida Fish and Wildlife Conservation Commission permit \#MTP-17-125A.

We conducted in-water captures of 20 green and 30 Kemp's ridley sea turtles by hand capture or using a dip net in the waters adjacent to the St. Martins Marsh Aquatic Preserve of Crystal River, Florida, USA ( $\left.28^{\circ} 50^{\prime} 24^{\prime \prime} \mathrm{N}, 82^{\circ} 45^{\prime} 00^{\prime \prime} \mathrm{W}\right)$, from June 12 to 17 , 2017. All turtles were reproductively immature, so sex could not be identified externally. For each turtle, we collected blood when the turtle was initially brought aboard the boat, then performed physical exams for an associated health study (McNally et al. 2020). As part of the physical exam, we inspected all turtles for FP by evaluating the total number of tumors and assigning a Balazs tumor score, which considers the size as well as the number of tumors (Balazs 1991; Table S1 in the Supplement at www. int-res.com/articles/suppl/n045p021_supp.pdf). Once the exam was completed and all samples were collected, each turtle was tagged to prevent resampling, and released from the side of the boat.

During the exams, we took an oral sample by gently swabbing the glottis of the turtle with a sterile cotton tipped applicator, using a sanitized bite block (Nylabone Products) to keep the mouth open. We then took a cloacal sample by gently inserting a cot- ton-tipped applicator approximately $2.5 \mathrm{~cm}$ into the cloaca and swabbing the interior mucosa. We placed swabs in individual cryovials that were immediately set on dry ice after collection. Upon return from the field, we transferred the samples to a cryogenic dewar of liquid nitrogen for storage until the field work was complete and samples could be transported to the laboratory. After arrival at the laboratory, ranging from 5 to $10 \mathrm{~d}$ after initial collection, we moved all samples to an ultra-low freezer $\left(-80^{\circ} \mathrm{C}\right)$ for storage until DNA extraction and sequencing could be completed.

To characterize the microbial community of the marine system, we also collected $1 \mathrm{l}$ of water at the site of the last turtle release on each day. Within $1 \mathrm{~h}$ after return from the field (approximately 1 to $2 \mathrm{~h}$ after collection), we filtered the water through a $0.22 \mu \mathrm{M}$ Sterivex ${ }^{\mathrm{TM}}$ filter and placed the filter in a labeled Whirl-Pak bag to store in the liquid nitrogen dewar. Additionally, we collected samples from the boat deck to assess it as a source of influence on the turtle samples. Upon return from the field each day, we collected swabs of the boat deck where the turtles were being held for exams using a sterile cottontipped applicator. We placed the swab in a cryovial and stored it in the liquid nitrogen dewar until the samples were transferred to an ultra-low freezer for long-term storage.

\subsection{DNA extraction}

We extracted DNA from swabs using a phenol: chloroform:isoamyl extraction protocol adapted from Mettel et al. (2010). We first suspended the swabs in PBL lysis buffer (water saturated phenol, disodium EDTA, sodium dodecyl sulfate, Tris- $\mathrm{HCl}, \mathrm{pH}$ 5.7) by vortexing and centrifuging. We removed the supernatant and placed it in a clean tube. After removal of the supernatant, we added TPM buffer $(50 \mathrm{mM}$ Tris, $\mathrm{pH} 7.0$, polyvinyl pyrrolidone, $\mathrm{MgCl}_{2}$ ) to the original tube with the swab; after vortexing and centrifuging, we then added the resulting supernatant to the tube with the first supernatant. We supplemented the combined supernatant with $800 \mu \mathrm{l}$ of a phenol:chloroform:isoamyl alcohol solution $(\mathrm{pH}$ $6.7+, 25: 24: 1)$ and centrifuged. We transferred the upper aqueous layer to a sterile tube and added 0.7 volumes of $100 \%$ isopropanol and 0.1 volumes of $3 \mathrm{M}$ sodium acetate. After centrifugation, the supernatant was decanted, we washed the pellet with $70 \%$ ethanol, and allowed it to air dry. We then resuspended the dried pellet in $50 \mu$ l nuclease-free 
water and stored it at $-80^{\circ} \mathrm{C}$ until amplification. We verified all DNA extracts by gel electrophoresis, including negative controls of unused sterile swabs, to ensure there was no contamination from supplies and solutions used in the extraction.

We extracted DNA from water samples using an adaptation of the manufacturer's guidelines for the MoBio PowerWater ${ }^{\circledR}$ Sterivex ${ }^{\mathrm{TM}}$ DNA Isolation Kit. We followed the manufacturer's instructions to generate the lysate (up through step 12 in the manufacturer's protocol). We removed the lysate with a $3 \mathrm{ml}$ syringe and added it to clean $2 \mathrm{ml}$ sterile tubes. To these tubes, we added $800 \mu \mathrm{l}$ of phenol:chloroform: isoamyl alcohol solution ( $\mathrm{pH} 6.7+, 25: 24: 1)$ and centrifuged. We transferred the upper aqueous layer to a sterile tube and added 0.7 volumes of $100 \%$ isopropanol and 0.1 volumes of $3 \mathrm{M}$ sodium acetate. After centrifugation, the supernatant was decanted, we washed the pellet with $70 \%$ ethanol, and allowed it to air dry. We then resuspended the dried pellet in $50 \mu \mathrm{l}$ nuclease-free water and stored it at $-80^{\circ} \mathrm{C}$ until amplification.

After verification, we amplified DNA extracts in triplicate using bacterial-specific (515F and 806R), uniquely barcoded, 16S rRNA primers containing adaptors for Illumina sequencing (Caporaso et al. 2012). Each $25 \mu \mathrm{l}$ PCR reaction contained $12.5 \mu \mathrm{l}$ Phusion Master Mix (Thermo Fisher), $0.5 \mu$ primers, $11 \mu \mathrm{l}$ diethylpyrocarbonate (DEPC) water, and $1 \mu \mathrm{l}$ of DNA. We verified the PCR product via gel electrophoresis, excised the target bands, and purified them using the QIAquick PCR Purification Kit (QIAGEN) following the manufacturer's protocol. We then quantified the purified product using a Qubit 2.0 Fluorometer (Thermo Fisher) and pooled it in equimolar concentrations. Sequencing was performed on the Illumina MiSeq platform with a paired-end V2 300 cycle kit.

\subsection{Data analysis}

Paired-end reads were demultiplexed using Illumina-utils version 2.0.2 (Eren et al. 2013). We performed quality filtering, merging of paired reads, and amplicon sequence variant (ASV) clustering using DADA2 version 1.12.1 (Callahan et al. 2016) in $\mathrm{R}$ version 3.6.1 ( $\mathrm{R}$ Core Team 2019). We assigned taxonomy using IDTAXA from the DECIPHER package version 2.12.0 (Murali et al. 2018) with the Silva Small Subunit (SSU) 132 training set for classification. We used the phyloseq package version 1.28.0 in $\mathrm{R}$ to perform diversity metric visualizations, heatmaps, and statistical tests (McMurdie \& Holmes 2013). We used Bray-Curtis distance metrics to evaluate the differences between each body site (oral and cloaca) for each species. We used principal coordinate analysis $(\mathrm{PCoA})$ to visualize variations in the microbial communities and we tested for significant differences using permutational multivariate analysis of variance (PERMANOVA). We calculated alpha diversity metrics and, since the data were not normally distributed (Shapiro-Wilks test, $\mathrm{p}=0.004$ ), we tested for significance using pairwise Wilcoxon tests, and tested for the significance of the influence of FP total tumors and Balazs score on the microbial communities using PERMANOVA for Bray-Curtis distance metrics and pairwise Wilcoxon test for alpha diversity metrics.

We identified important taxa between the microbial communities of each species using the DESeq2 package version 1.24.0 (Love et al. 2014), which identifies features that are differentially abundant across samples. We defined the core microbiome as ASVs present in a minimum of $90 \%$ of the turtle samples specific to each body site and each species, which we identified using the microbiome package version 1.6.0 (Lahti \& Shetty 2017).

All sequencing data generated for this study are available at NCBI's Sequence Read Archive, under BioProject accession number: PRJNA678525.

\section{RESULTS}

\subsection{Sample data}

We successfully examined a total of $30 \mathrm{Kemp}$ 's ridley turtles and 20 green turtles in Crystal River, Florida, USA. Twenty-nine of the Kemp's ridley turtles were similar in size with a mean straight standard carapace length (SSCL) of $46.7 \mathrm{~cm}$ and a mean weight of $15.4 \mathrm{~kg}$, while 1 was smaller with an SSCL of $24.6 \mathrm{~cm}$ and weight of $2.2 \mathrm{~kg}$ (Table 1). We captured all Kemp's ridley turtles by hand except for the smallest animal, which was captured with a dip net. All 30 Kemp's ridley turtles appeared clinically healthy with no visible injuries (McNally et al. 2020). We captured 5 green turtles by hand and the remaining 15 were captured with a dip net. The mean SSCL of the green turtles was $38.0 \mathrm{~cm}$ and the mean weight was $7.0 \mathrm{~kg}$ (Table 1). No Kemp's ridley turtles had visible tumors consistent with FP, but we observed a high prevalence of FP in the green turtles with $90 \%$ of captured green turtles having visible tumors (total tumors mean 17.2, Balazs score mean 1.6, Table 1). 
Table 1. Health assessment information and morphometric data of sampled sea turtles separated by species. SSCL: straight standard carapace length

\begin{tabular}{|lccccc|}
\hline & Water temp $\left({ }^{\circ} \mathrm{C}\right)$ & Weight $(\mathrm{kg})$ & SSCL $(\mathrm{cm})$ & Total tumors & Balazs score \\
\hline Kemp's ridley $(\mathrm{n}=30)$ Mean $\pm \mathrm{SD}$ & $28.5 \pm 1.4$ & $15.0 \pm 4.8$ & $45.9 \pm 6.2$ & 0 & 0 \\
Range (min-max) & $(26.6-30.8)$ & $(2.2-23.5)$ & $(24.6-53.6)$ & & \\
Green (n = 20) Mean \pm SD & $28.6 \pm 1.0$ & $7.0 \pm 2.2$ & $38.0 \pm 4.0$ & $17.2 \pm 14.0$ & $1.6 \pm 1.0$ \\
Range (min-max) & $(26.7-30.8)$ & $(4.1-13.1)$ & $(31-47)$ & $(0-38)$ & $(0-3)$ \\
\hline
\end{tabular}

\subsection{The oral and cloacal microbiome}

Across all samples, sequencing of the 16S rRNA gene resulted in 2727027 reads after joining pairedends and quality filtering, which included the removal of chimeras, singletons, chloroplasts, mitochondrial DNA, and archaea. Out of 107 samples, 1 sample (a cloaca sample from a green turtle) did not yield enough sequences to be included and was removed from downstream analyses. The mean sequence counts per sample was 25727 (median 23173) and the range was 7306 to 88976 counts per sample. These sequences were assigned to 1335 unique ASVs (a measure of sequence similarity that can be used to differentiate taxa) across 181 different families.

Kemp's ridley turtles had significantly higher Shannon diversity at each body site compared to green turtles for both oral and cloaca samples (Fig. 1; Wilcoxon, $\mathrm{p}<0.001)$. Oral samples had higher Shannon diversity than cloaca samples in Kemp's ridley turtles (Wilcoxon, $\mathrm{p}<0.001$ ), but the Shannon diversity was similar between oral and cloaca samples of green turtles (Fig. 1). The oral samples of Kemp's ridley turtles also had higher Shannon diversity than the water samples, which had a mean of 2.8 and standard deviation of 0.5 (Wilcoxon, $\mathrm{p}<0.001$ ). The water samples were similar in diversity to the Kemp's ridley turtle cloaca samples, while higher in diversity compared to green turtle oral and cloaca samples (Wilcoxon, $\mathrm{p}=0.029$ ). The boat deck samples also had significantly higher Shannon diversity than both green oral and cloaca samples (Wilcoxon, $\mathrm{p}=0.029$ ).

The oral and cloacal microbial communities were distinct from each other within each species and the structure of the microbial communities was significantly different between each body site and species based on Bray-Curtis dissimilarity (Fig. 2). The oral microbiome of Kemp's ridley turtles was dominated by bacteria in the family Flavobacteriaceae (Fig. 3), with a mean abundance of $34.8 \%$, followed by Arcobacteraceae (11.6\%) and Rhodobacteraceae $(8.7 \%)$, while the green turtle oral cavity was dominated by Pasteurellaceae (44.8\%), followed by Arco- bacteraceae (15.6\%), Campylobacteraceae (9.9\%), and Desulfobulbaceae (9.2\%). The cloaca samples of both turtle species had high proportions of Neisseriaceae, though they were relatively more abundant in the green turtles $(29.2 \%)$ than in the Kemp's ridley turtles $(10.4 \%)$. Green turtle cloaca samples also had a high percentage of the families Arcobacteraceae $(14.7 \%)$ and Desulfobulbaceae (11.4\%). In addition to the Neisseriaceae, Kemp's ridley cloaca samples had a large proportion of Cardiobacteriaceae (16.5\%) and Flavobacteriaceae (15.5\%) (Fig. 3).

Water and deck samples were distinct from all the turtle samples (Fig. S1 in the Supplement). Water sam-

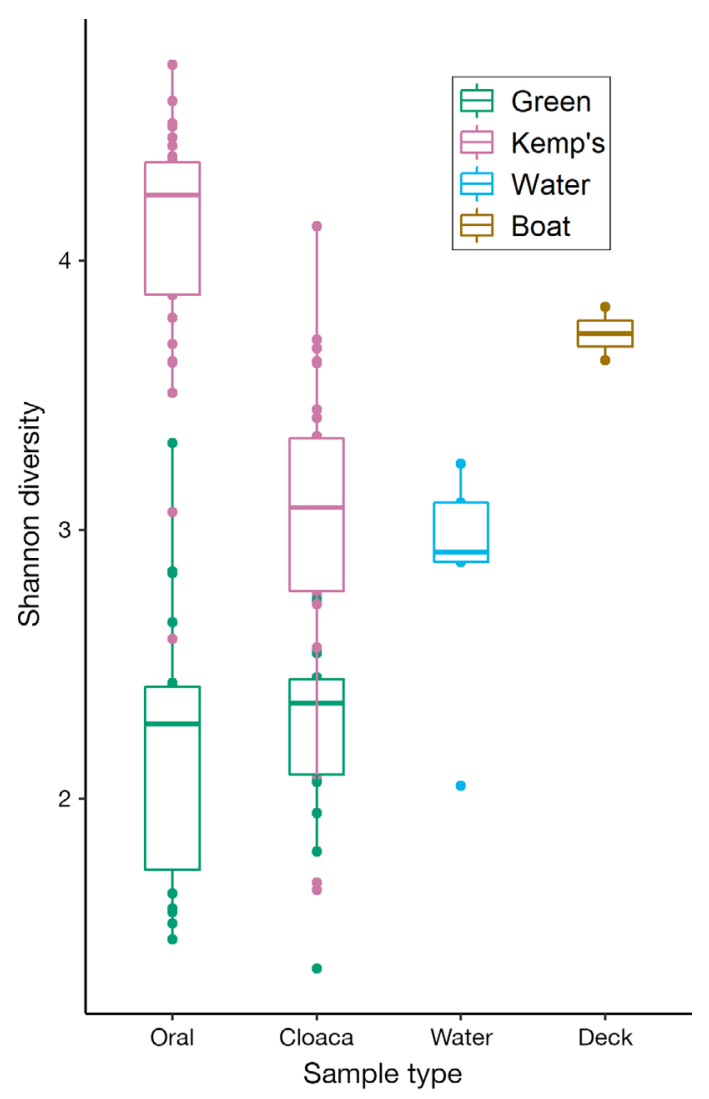

Fig. 1. Shannon diversity as a boxplot (median, 1st and 3rd quartiles, min-max, and outliers shown as dots) with sample types on $x$-axis. Colors indicate the turtle species (green or Kemp's ridley turtles) or sample location (water or boat deck) 


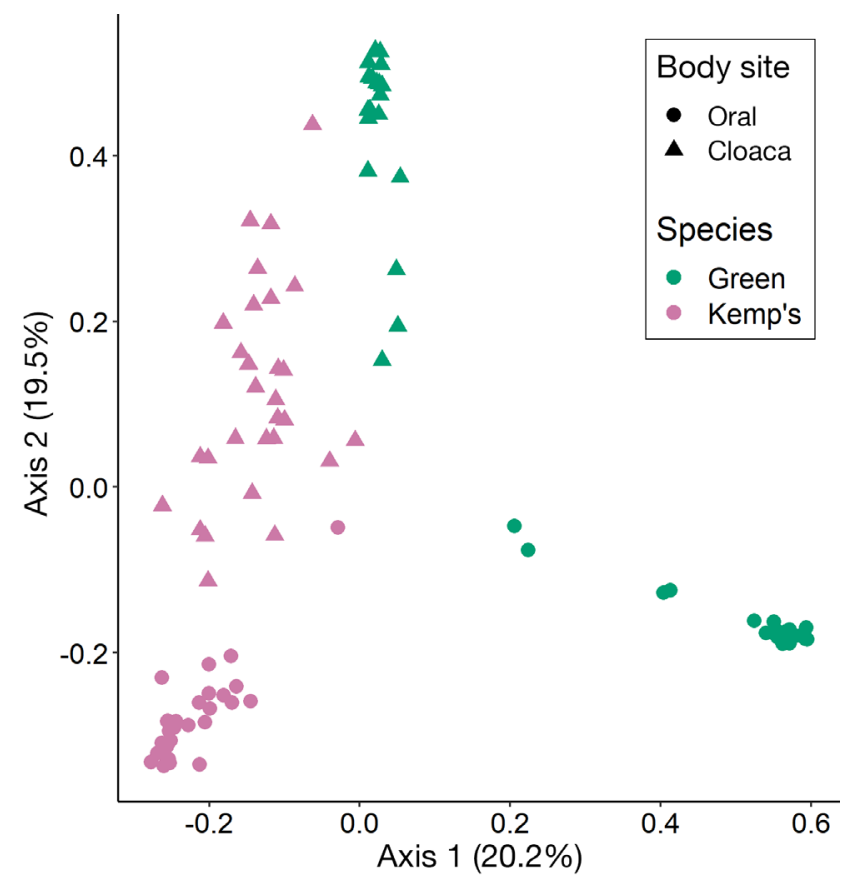

Fig. 2. Principal coordinate analysis (PCoA) plots of samples based on Bray-Curtis dissimilarity

ples were dominated by bacteria in the families Rhodobacteraceae (33.9\%), Flavobacteriaceae (19.4\%), Thioglobaceae (15.5\%), and Litoricolaceae (9.2\%). The boat deck, which was in contact with the turtle skin, sea water, and humans, had highest proportions of Idiomarinaceae $(24.0 \%)$, Marinobacteraceae (19.1\%), Halomonadaceae (12.1\%), and Alteromonadaceae $(9.8 \%)$. Since we did not see discrete signatures of these taxa in the turtle microbiomes, we focused only on turtles for the remaining analyses.

We found 204 ASVs with significant differences in abundance between the green turtle and Kemp's ridley turtle oral samples, and the cloaca samples had 108 significantly different ASVs between the 2 species. The oral cavity ASVs that had highest relative abundance in the Kemp's ridley turtles compared to green turtles largely consisted of bacteria from the families Arcobacteraceae and Flavobacteriaceae. ASVs that were more abundant in green turtles were similarly from the family Arcobacteraceae, but also included ASVs in the families Desulfobulbaceae and Campylobacteraceae (Table 2, Fig. 4A). ASVs with the largest difference in cloaca samples between species were from the families Arcobacteraceae, Desulfobulbaceae, Leptotrichiaceae, and Campylobacteraceae, which were more abundant in green turtles; whereas ASVs from Rhodocyclaceae, Cardiobacteriaceae, Campylobacteraceae, Endozoicomonadaceae, Tannerellaceae, and Flavobacteriaceae were sig- nificantly more abundant in Kemp's ridley turtles (Table 2, Fig. 4B).

\subsection{Core microbiome analysis}

We found only 4 ASVs shared in $90 \%$ of oral samples across both turtle species and no ASVs shared across both species in the cloaca samples (Fig. 5). Among the individual body sites within each species, however, there were several ASVs that had greater than 90\% prevalence (Table 3, Fig. 5). The oral samples of green turtles had 11 ASVs present in at least $90 \%$ of the samples and the green turtle cloacal microbial community also shared 11 ASVs that were in at least $90 \%$ of all samples. The Kemp's ridley oral samples had 23 ASVs that fit our definition of a core microbiome, whereas the cloaca samples only had 2 core ASVs.

\subsection{Fibropapillomatosis and the turtle microbiome}

FP tumors were present in $90 \%$ of the green turtles captured. Turtles had a total tumor mean of 17.2 (range 0 to 38) and a Balazs score mean of 1.6 (range 0 to 3). Microbial communities were significantly different for green turtle cloaca samples as a function of total tumors (PERMANOVA, $\mathrm{p}=0.008$ ), though they were not significantly different in oral samples (Fig. 6; PERMANOVA, $\mathrm{p}=0.175$ ). There was no significant difference in Shannon diversity among green turtles in either the oral or cloacal microbial communities based on Balazs score. We also examined the change in community as a function of Balazs score (Fig. 7). The turtle with the highest Balazs score (most severe level of infection) had higher relative abundances of Fusobacteriaceae and Acidaminococcaceae, and lower abundances of Arcobacteraceae in the oral sample compared to those from the other Balazs scores. In general, the turtles with a lower Balazs score and total number of tumors had more Vibrionaceae and Arcobacteraceae in cloaca samples compared to the higher scores and total tumors.

\section{DISCUSSION}

Variation in microbial communities within a species can be caused by the organism's local environment, life stage, and diet, among other things (McFall-Ngai et al. 2013, Keenan \& Elsey 2015, Colston \& Jackson 2016). In this study, we characterized the microbiome of juvenile Kemp's ridley and green tur- 


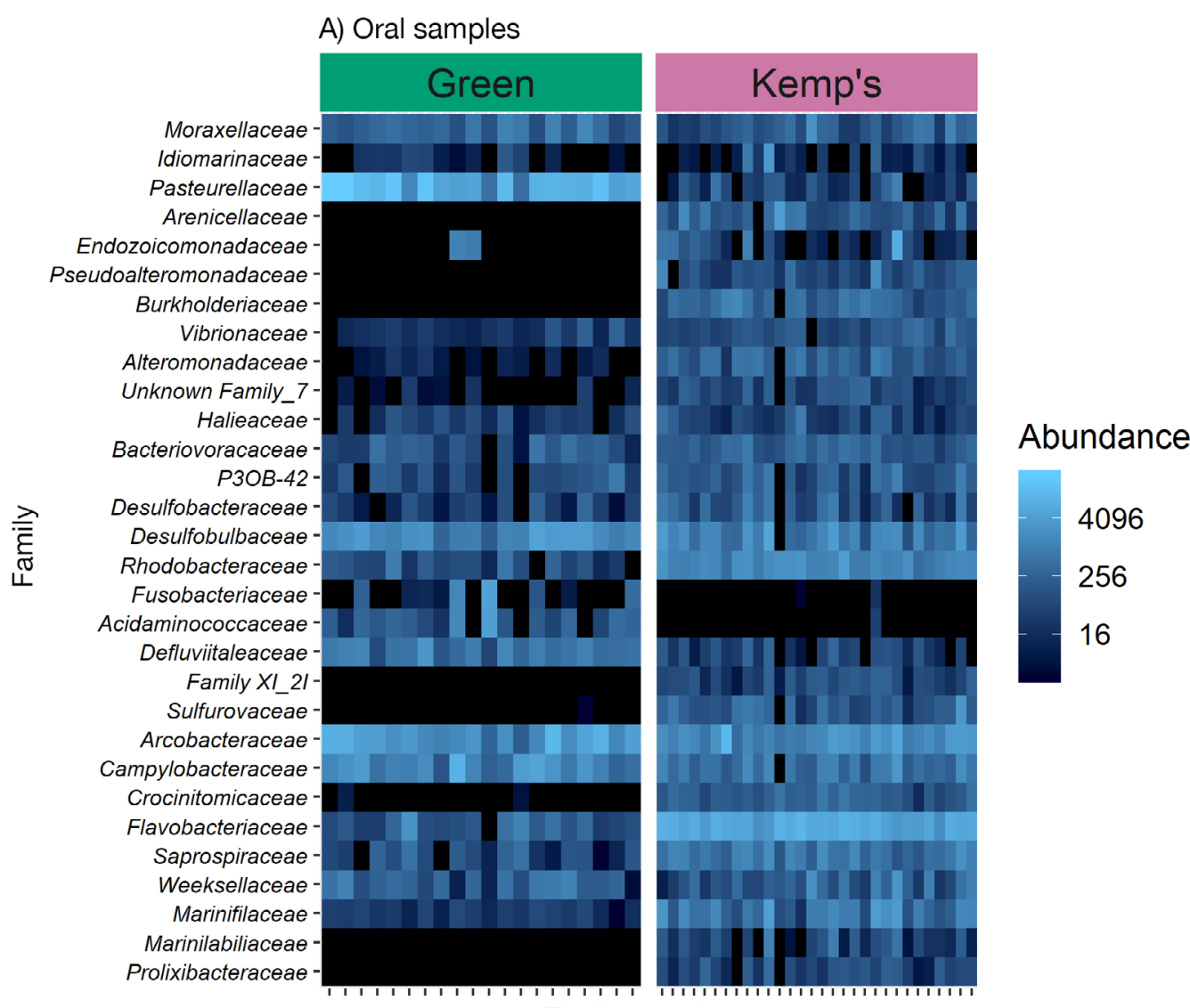

Turtle samples

B) Cloaca samples

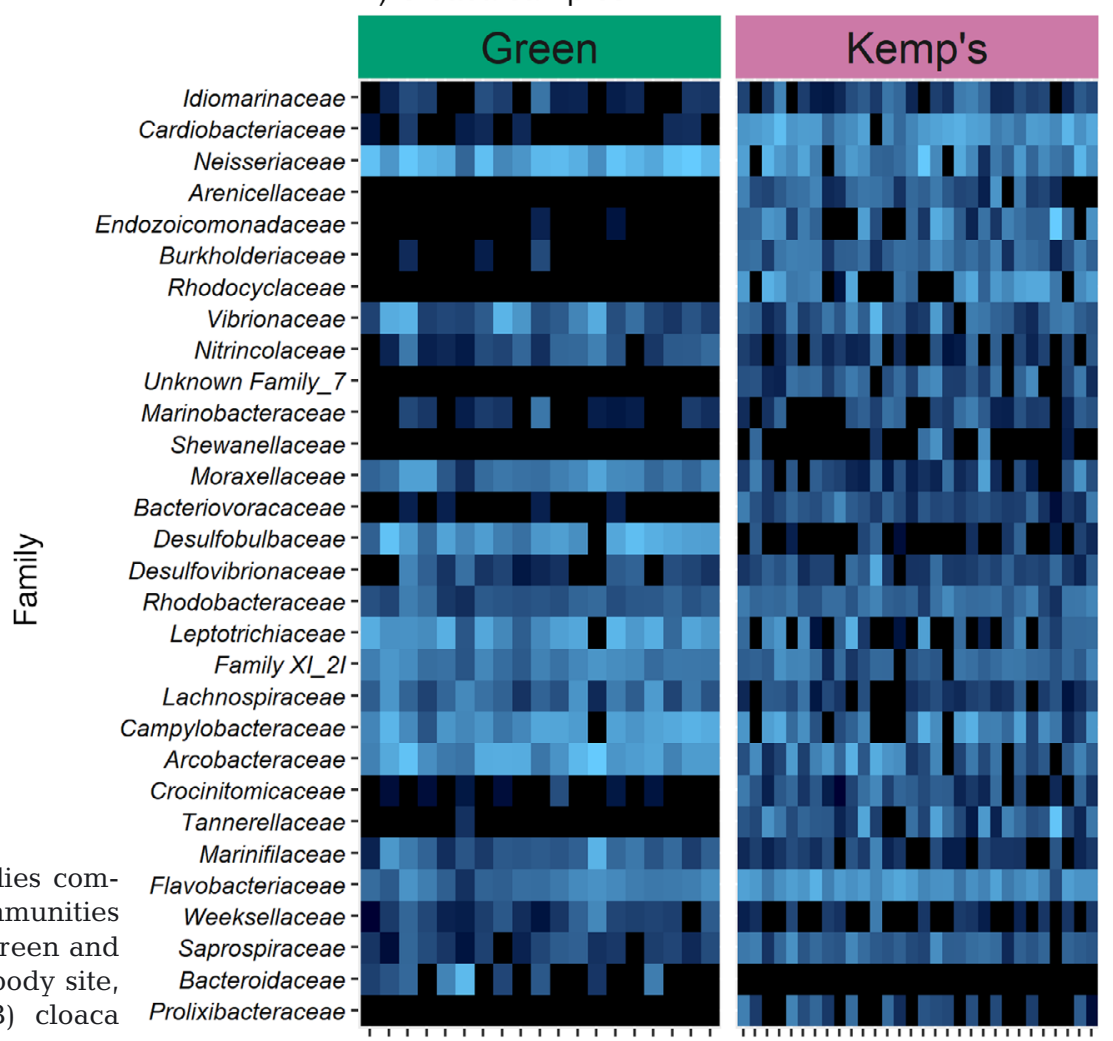

Turtle samples

Fig. 3. The top 30 families composing the bacterial communities of each turtle species (green and Kemp's ridley) at each body site (A) oral cavity and (B) cloaca

A) Oral samples 
Table 2. The top 20 amplicon sequence variants (ASVs) that displayed the largest difference in relative abundance (\%) and standard deviation (SD) between green and Kemp's ridley turtles in both oral and cloaca samples. NA: not available

\begin{tabular}{|c|c|c|c|c|c|c|c|}
\hline \multirow[t]{2}{*}{ ASV } & \multicolumn{3}{|c|}{ - Taxonomy } & \multicolumn{2}{|l|}{ Green } & \multicolumn{2}{|c|}{- Kemp's } \\
\hline & Order & Family & Genus & $\begin{array}{c}\text { Relative } \\
\text { abund. (\%) }\end{array}$ & $\begin{array}{l}\mathrm{SD} \\
(\%)\end{array}$ & $\begin{array}{c}\text { Relative } \\
\text { abund. (\%) }\end{array}$ & $\begin{array}{l}\text { SD } \\
(\%)\end{array}$ \\
\hline \multicolumn{8}{|l|}{ Oral } \\
\hline ASV1016 & Campylobacterales & Campylobacteraceae & Campylobacter & 2.25 & 3.45 & 0.05 & 0.10 \\
\hline ASV627 & Clostridiales & Defluviitaleaceae & UCG-011 & 1.94 & 1.72 & 0.00 & 0.00 \\
\hline ASV709 & Desulfobacterales & Desulfobulbaceae & NA & 2.44 & 3.62 & 0.00 & 0.01 \\
\hline ASV272 & Campylobacterales & Arcobacteraceae & Arcobacter & 9.31 & 8.76 & 0.00 & 0.00 \\
\hline ASV197 & Campylobacterales & Campylobacteraceae & Campylobacter & 6.59 & 9.79 & 0.00 & 0.00 \\
\hline ASV492 & Campylobacterales & Arcobacteraceae & Arcobacter & 2.75 & 2.10 & 0.00 & 0.00 \\
\hline ASV452 & Desulfobacterales & Desulfobulbaceae & Desulforhopalus & 2.32 & 2.03 & 0.00 & 0.00 \\
\hline ASV560 & Desulfobacterales & Desulfobulbaceae & NA & 1.72 & 1.55 & 0.00 & 0.00 \\
\hline ASV545 & Bacteroidales & Marinifilaceae & Marinifilum & 0.00 & 0.00 & 1.48 & 1.85 \\
\hline ASV279 & Flavobacteriales & Flavobacteriaceae & NA & 0.00 & 0.00 & 1.17 & 1.49 \\
\hline ASV924 & Flavobacteriales & Flavobacteriaceae & NA & 0.01 & 0.02 & 1.75 & 1.43 \\
\hline ASV1291 & Flavobacteriales & Flavobacteriaceae & NA & 0.00 & 0.00 & 1.55 & 1.68 \\
\hline ASV377 & Campylobacterales & Arcobacteraceae & Arcobacter & 0.00 & 0.00 & 3.00 & 8.98 \\
\hline ASV43 & Flavobacteriales & Flavobacteriaceae & NA & 0.00 & 0.00 & 2.55 & 2.45 \\
\hline ASV467 & Bacteroidales & Marinifilaceae & Marinifilum & 0.00 & 0.00 & 1.65 & 1.51 \\
\hline ASV917 & Flavobacteriales & Flavobacteriaceae & NA & 0.00 & 0.00 & 3.55 & 4.27 \\
\hline ASV619 & Betaproteobacteriales & Burkholderiaceae & NA & 0.00 & 0.00 & 1.96 & 2.01 \\
\hline ASV1111 & Flavobacteriales & Flavobacteriaceae & NA & 0.00 & 0.00 & 5.23 & 5.15 \\
\hline ASV1104 & Campylobacterales & Arcobacteraceae & Arcobacter & 0.00 & 0.00 & 2.95 & 2.45 \\
\hline ASV508 & Campylobacterales & Arcobacteraceae & Arcobacter & 0.00 & 0.00 & 2.73 & 2.06 \\
\hline \multicolumn{8}{|l|}{ Cloaca } \\
\hline ASV166 & Campylobacterales & Arcobacteraceae & Arcobacter & 6.99 & 8.28 & 0.15 & 0.70 \\
\hline ASV1302 & Campylobacterales & Arcobacteraceae & Arcobacter & 3.38 & 4.95 & 0.21 & 1.12 \\
\hline ASV411 & Campylobacterales & Arcobacteraceae & Arcobacter & 3.78 & 4.06 & 0.01 & 0.05 \\
\hline ASV1068 & Desulfobacterales & Desulfobulbaceae & NA & 7.53 & 8.04 & 0.03 & 0.18 \\
\hline ASV721 & Desulfobacterales & Desulfobulbaceae & NA & 3.53 & 3.97 & 0.10 & 0.24 \\
\hline ASV313 & Fusobacteriales & Leptotrichiaceae & NA & 9.47 & 8.58 & 2.16 & 4.27 \\
\hline ASV517 & Campylobacterales & Campylobacteraceae & Campylobacter & 9.64 & 6.46 & 0.83 & 1.23 \\
\hline ASV726 & Cardiobacteriales & Cardiobacteriaceae & NA & 0.00 & 0.00 & 2.29 & 3.76 \\
\hline ASV222 & Clostridiales & Family XI_2I & Fusibacter & 0.00 & 0.00 & 1.09 & 1.43 \\
\hline ASV514 & Betaproteobacteriales & Rhodocyclaceae & NA & 0.00 & 0.00 & 7.89 & 9.04 \\
\hline ASV1245 & Campylobacterales & Campylobacteraceae & Campylobacter & 0.03 & 0.10 & 4.70 & 6.53 \\
\hline ASV935 & Flavobacteriales & Flavobacteriaceae & NA & 0.00 & 0.00 & 1.15 & 1.82 \\
\hline ASV1104 & Campylobacterales & Arcobacteraceae & Arcobacter & 0.00 & 0.00 & 1.64 & 3.45 \\
\hline ASV1164 & Cardiobacteriales & Cardiobacteriaceae & Cardiobacterium & 0.00 & 0.01 & 12.39 & 11.49 \\
\hline ASV798 & Flavobacteriales & Flavobacteriaceae & NA & 0.00 & 0.00 & 1.78 & 2.33 \\
\hline ASV1046 & Oceanospirillales & Endozoicomonadaceae & Endozoicomonas & 0.00 & 0.01 & 4.97 & 9.56 \\
\hline ASV1310 & Bacteroidales & Tannerellaceae & Macellibacteroides & 0.01 & 0.02 & 3.43 & 6.69 \\
\hline ASV882 & Flavobacteriales & Flavobacteriaceae & NA & 0.00 & 0.00 & 2.75 & 3.56 \\
\hline ASV1072 & Arenicellales & Arenicellaceae & Arenicella & 0.00 & 0.00 & 1.52 & 2.69 \\
\hline ASV619 & Betaproteobacteriales & Burkholderiaceae & NA & 0.00 & 0.00 & 1.19 & 1.36 \\
\hline
\end{tabular}

tles from coastal western Florida. Several green sea turtle studies from various regions of the world have examined microbial communities of either cloaca or fecal samples (Ahasan et al. 2017, Price et al. 2017, Campos et al. 2018). Price et al. (2017) characterized the juvenile green turtle cloacal microbiome from different regions of Florida and from 2 habitats (pelagic and neritic). The turtles from the coastal habitat were most similar to the turtles in our study which were also collected from coastal systems. The families of Neisseriaceae, Arcobacteraceae, Campylobacteraceae, and Desulfobulbaceae were relatively abundant in the green turtles of both studies. By contrast, fecal samples collected from wild green turtles from Australia and Brazil had microbiomes dominated by the families Bacteroidiaceae, Lachnospiraceae, Clostridiaceae, and Porphyromonadaceae (Ahasan et al. 2017, Campos et al. 2018). Although present in low abundances in the turtles from Florida, they were not dominant in the cloaca samples of juvenile 
A) Oral samples

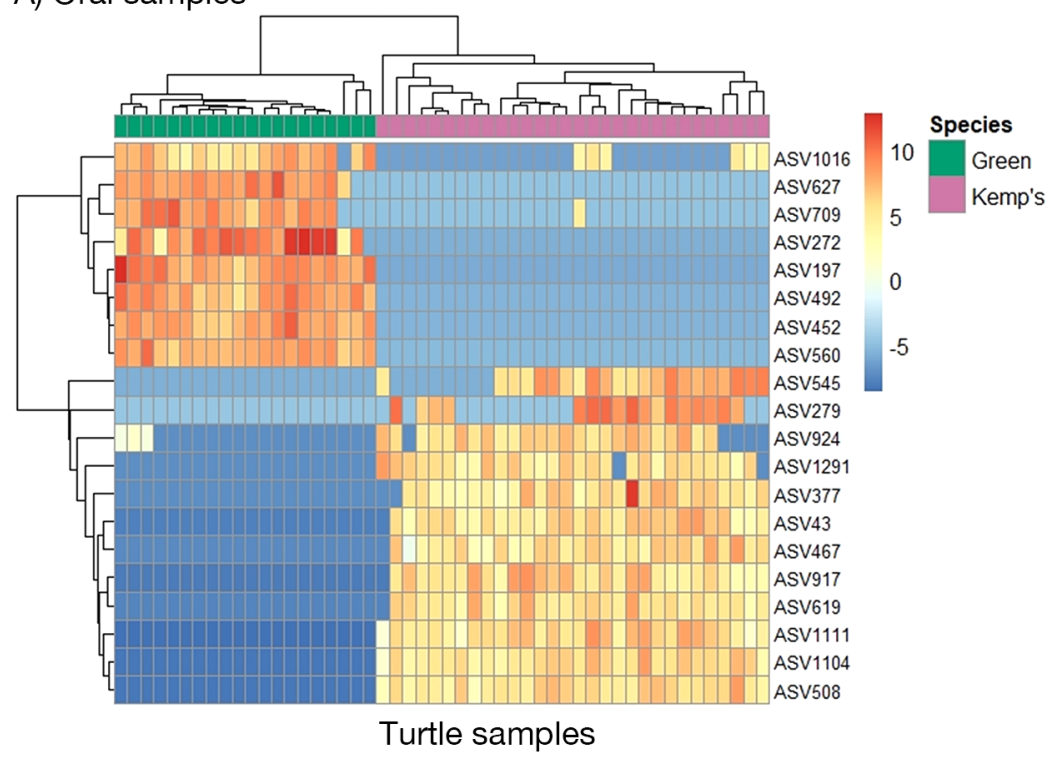

B) Cloaca samples

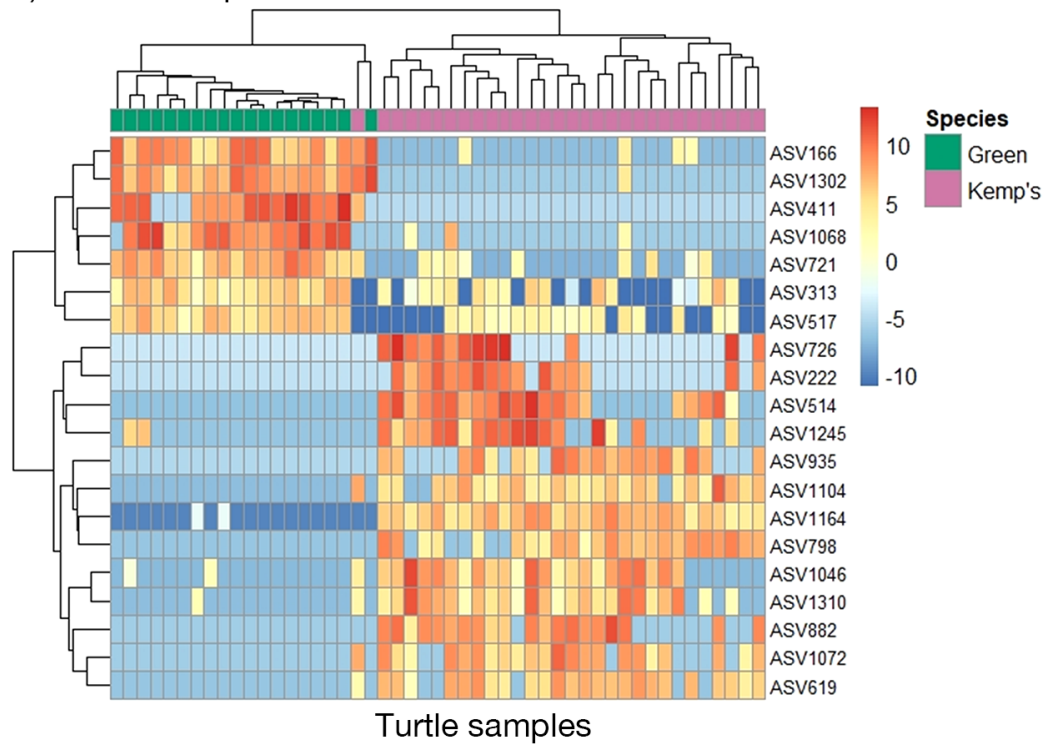

Fig. 4. The top 20 amplicon sequence variants (ASVs) that displayed the largest difference in relative abundance between Kemp's ridley turtles and green turtles for (A) oral samples and (B) cloaca samples. Counts were normalized using variance stabilizing transformation. Taxonomy of the ASVs is shown in Table 2

green turtles in our study. This could be due to differences in location, life stage, prevalence of FP, or the section of the gastrointestinal tract that was sampled. For example, in alligators, fecal samples were significantly different from those taken from other parts of the gastrointestinal (GI) tract (Keenan et al. 2013, Keenan \& Elsey 2015). Alligators had fecal samples primarily composed of the phylum Fusobacteria and intestinal samples with higher proportions of Firmi- cutes, which shifted to predominantly Proteobacteria during the winter months (Keenan et al. 2013). Sections of the GI tract of green turtles also differ from each other, with anaerobes more likely to survive in the anterior sections (Ahasan et al. 2020). Thus, the differences between the cloacal communities observed in our study and the other sections of the GI and fecal samples collected in other studies could be due to real regional differences in the microbiome or could be a result of innate differences between GI, cloacal, and fecal microbiomes.

This is the first study to our knowledge to report wild Kemp's ridley turtle microbiomes at this life stage. The only other published study including wild healthy Kemp's ridley turtle microbial communities involves colonic swabs from adult nesting turtles in Mexico (Scheelings et al. 2020a). The adult samples were predominately composed of the order Campylobacterales, which were also present in our Kemp's ridley cloaca samples, though the samples diverged at the family level. Although there are a variety of differences in these studies (cloacal swab depth, geography), the dissimilarities in microbial communities are also emphasized by the different life stages. Samuelson et al. (2020) recently examined the fecal microbiome of rehabilitated juvenile Kemp's ridley turtles and found the class Clostridia was most abundant in the fecal samples of turtles in short-term rehabilitation. Although our Kemp's ridley cloaca samples had a small proportion of Clostridia in the microbiome, the dominant classes were Gammaproteobacteria and Bacteroidia. The differences between the rehabilitated and wild healthy Kemp's ridley turtle microbial communities are not limited to the type of sampling (feces vs. cloaca); other variables including environment (i.e. rehabilitation tanks, geography), health condition, medications, and available diet could also explain these differences.

Thus far, studies of microbial communities in sea turtles have focused on fecal or cloacal microbiomes. 


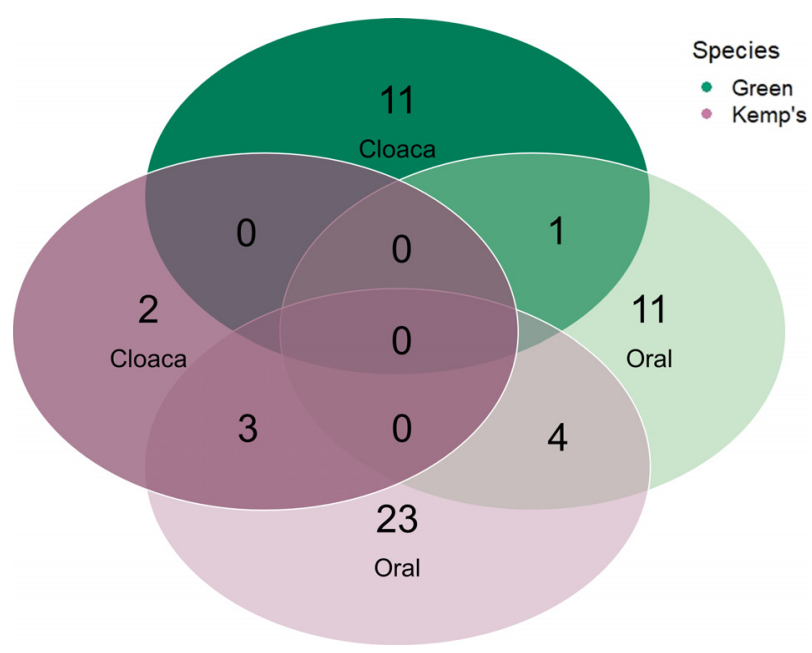

Fig. 5. Number of amplicon sequence variants (ASVs) shared in $90 \%$ of samples based on turtle species (green and Kemp's ridley) and body site

Our study is unique in that we also characterized the microbiome of the oral cavity. The importance of the oral microbiome is unknown in many animals. The oral microbes of other species of reptiles, alligator and Komodo dragon have been evaluated. In the alligator, the oral samples had higher alpha diversity than the lower GI tract (Keenan et al. 2013), consistent with what we observed with the Kemp's ridley turtles (Fig. 1). The salivary microbiome of Komodo dragons similarly had higher Shannon diversity than fecal samples (Hyde et al. 2016). A similar pattern has been seen in marine mammals, with samples collected from the oral cavity having higher alpha diversity than rectal samples (Bik et al. 2016). This is thought to be due to greater interaction with transient microbes from the environment entering the oral cavity. However, we did not observe this pattern in green turtles. In fact, green turtles had lower alpha diversity at both body sites compared to the surrounding water, indicating that the water column microbes had little influence on the green turtle microbiome. Kemp's ridley turtles also had higher Shannon diversity than the green turtles at each body site. This result was unexpected because other studies, particularly in mammals, have indicated that herbivores have higher alpha diversity compared to carnivores, possibly due to the need for more diverse bacteria to effectively ferment the plant cell wall polysaccharides (Ley et al. 2008a). Two species of herbivorous iguanas, however, had different levels of alpha diversity, which may be due to the complexity of the specific vegetation being consumed (Hong et al. 2011). It is possible that Kemp's ridley turtles still require a diverse microbial community to digest their hardshelled food items.

We sampled Kemp's ridley and green turtles from the same environment, ruling out location-specific environmental variables as the cause of taxonomic differences in microbial communities. Although there are no previous studies of Kemp's ridley turtles at this life stage for comparison, they are clearly different from the sympatric green turtles. In the oral and cloaca samples, Campylobacter sp. and Arcobacter sp., both in the order Campylobacterales, comprised the biggest differences between the turtle species, with both genera having multiple ASVs that were differentially abundant in one or the other turtle species (Table 2). For example, Kemp's ridley turtles had 3 ASVs from the Arcobacter genus that were more abundant than green turtle oral or cloaca samples, but different ASVs of this genus were more abundant in green turtles. This could be due to the diverse bacteria from this genus found in the environment, including food items, of the sea turtles. Arcobacter are common in sea water, oysters, and even sewage, and they have also been found in intestine samples and feces of farm animals (Collado \& Figueras 2011). Although they are associated with disease in some farm animals, they are more commonly found in healthy animals (Collado \& Figueras 2011).

Multiple ASVs of the family Flavobacteriaceae were more abundant in Kemp's ridley oral or cloaca samples compared to those of green turtles. Flavobacteriaceae are common in marine environments, particularly in shellfish (Jooste \& Hugo 1999). Flavobacteriaceae are in many marine mammal microbiomes, including oral and gastric samples from sea lions (Bik et al. 2016) and humpback whale respiratory vapor and skin (Apprill et al. 2014, 2017). Although common to the marine environment, we found this family of bacteria only in the Kemp's ridley turtles, not green turtles. Thus, they may be essential to Kemp's ridley turtles, potentially playing a role in digestion, or they could be transiently carried to the Kemp's ridley turtles from a particular food item. In humpback whales, the Flavobacteriaceae on the skin are thought to provide a protective function by predating on other types of bacteria (Apprill et al. 2014), providing another possible role for this family of bacteria in Kemp's ridley turtles. It appears unlikely that the Flavobacteriaceae found in Kemp's ridley turtles are pathogenic, as they were highly prevalent and all turtles in this study appeared healthy.

Cardiobacteriaceae is another family of bacteria that we found in high abundance in Kemp's ridley 
Table 3. Core microbiome. The amplicon sequence variants (ASVs) that are shared among turtle species and body sites with taxonomy and prevalence (proportion of samples in which the ASV is found) across the indicated sample type. Core was defined as minimum prevalence of $0.90(90 \%)$. NA: not available

\begin{tabular}{|c|c|c|c|c|}
\hline Turtle species & Body site & ASV & Order, Family, Genus & Prevalence \\
\hline \multirow[t]{11}{*}{ Green } & \multirow[t]{11}{*}{ Cloaca } & ASV19 & Clostridiales, Lachnospiraceae, NA & 1.00 \\
\hline & & ASV40 & Pseudomonadales, Moraxellaceae, Moraxella & 0.95 \\
\hline & & ASV166 & Campylobacterales, Arcobacteraceae, Arcobacter & 1.00 \\
\hline & & ASV313 & Fusobacteriales, Leptotrichiaceae, NA & 0.95 \\
\hline & & ASV381 & Flavobacteriales, Flavobacteriaceae, NA & 1.00 \\
\hline & & ASV517 & Campylobacterales, Campylobacteraceae, Campylobacter & 0.95 \\
\hline & & ASV721 & Desulfobacterales, Desulfobulbaceae, NA & 0.95 \\
\hline & & ASV761 & Clostridiales, Family XI_2I, Fusibacter & 0.95 \\
\hline & & ASV978 & Clostridiales, Family XI_2I, Fusibacter & 1.00 \\
\hline & & ASV1130 & Betaproteobacteriales, Neisseriaceae, Snodgrassella & 1.00 \\
\hline & & ASV1302 & Campylobacterales, Arcobacteraceae, Arcobacter & 1.00 \\
\hline \multirow[t]{2}{*}{ Kemp's ridley } & \multirow[t]{2}{*}{ Cloaca } & ASV917 & Flavobacteriales, Flavobacteriaceae, NA & 0.93 \\
\hline & & ASV1164 & Cardiobacteriales, Cardiobacteriaceae, Cardiobacterium & 0.97 \\
\hline \multirow[t]{11}{*}{ Green } & \multirow[t]{11}{*}{ Oral } & ASV197 & Campylobacterales, Campylobacteraceae, Campylobacter & 1.00 \\
\hline & & ASV272 & Campylobacterales, Arcobacteraceae, Arcobacter & 0.95 \\
\hline & & ASV439 & Vibrionales, Vibrionaceae, NA & 0.95 \\
\hline & & ASV452 & Desulfobacterales, Desulfobulbaceae, Desulforhopalus & 1.00 \\
\hline & & ASV492 & Campylobacterales, Arcobacteraceae, Arcobacter & 1.00 \\
\hline & & ASV560 & Desulfobacterales, Desulfobulbaceae, NA & 1.00 \\
\hline & & ASV584 & Clostridiales, Defluviitaleaceae, NA & 1.00 \\
\hline & & ASV669 & Campylobacterales, Arcobacteraceae, Arcobacter & 0.95 \\
\hline & & ASV1016 & Campylobacterales, Campylobacteraceae, Campylobacter & 0.95 \\
\hline & & ASV1161 & Pasteurellales, Pasteurellaceae, Phocoenobacter & 1.00 \\
\hline & & ASV1281 & Pseudomonadales, Moraxellaceae, NA & 1.00 \\
\hline \multirow[t]{23}{*}{ Kemp's ridley } & \multirow[t]{23}{*}{ Oral } & ASV41 & Campylobacterales, Campylobacteraceae, Campylobacter & 0.97 \\
\hline & & ASV43 & Flavobacteriales, Flavobacteriaceae, NA & 0.97 \\
\hline & & ASV187 & Campylobacterales, Campylobacteraceae, Campylobacter & 0.93 \\
\hline & & ASV377 & Campylobacterales, Arcobacteraceae, Arcobacter & 0.93 \\
\hline & & ASV434 & Desulfobacterales, Desulfobulbaceae, Desulforhopalus & 0.97 \\
\hline & & ASV467 & Bacteroidales, Marinifilaceae, Marinifilum & 0.97 \\
\hline & & ASV499 & Campylobacterales, Sulfurovaceae, Sulfurovum & 0.97 \\
\hline & & ASV508 & Campylobacterales, Arcobacteraceae, Arcobacter & 1.00 \\
\hline & & ASV546 & Flavobacteriales, Flavobacteriaceae, NA & 0.93 \\
\hline & & ASV575 & Flavobacteriales, Flavobacteriaceae, Maritimimonas & 1.00 \\
\hline & & ASV619 & Betaproteobacteriales, Burkholderiaceae, NA & 0.97 \\
\hline & & ASV687 & Flavobacteriales, Weeksellaceae, NA & 0.93 \\
\hline & & ASV798 & Flavobacteriales, Flavobacteriaceae, NA & 0.93 \\
\hline & & ASV834 & Chitinophagales, Saprospiraceae, NA & 0.97 \\
\hline & & ASV843 & Myxococcales, $P 3 O B-42$, NA & 0.97 \\
\hline & & ASV917 & Flavobacteriales, Flavobacteriaceae, NA & 0.97 \\
\hline & & ASV935 & Flavobacteriales, Flavobacteriaceae, NA & 0.93 \\
\hline & & ASV1104 & Campylobacterales, Arcobacteraceae, Arcobacter & 1.00 \\
\hline & & ASV1111 & Flavobacteriales, Flavobacteriaceae, NA & 1.00 \\
\hline & & ASV1112 & Bdellovibrionales, Bacteriovoracaceae, Peredibacter & 0.97 \\
\hline & & ASV1281 & Pseudomonadales, Moraxellaceae, NA & 0.97 \\
\hline & & ASV1291 & Flavobacteriales, Flavobacteriaceae, NA & 0.93 \\
\hline & & ASV1305 & Flavobacteriales, Flavobacteriaceae, Maritimimonas & 0.97 \\
\hline \multirow{4}{*}{$\begin{array}{l}\text { Green \& } \\
\text { Kemp's ridley }\end{array}$} & \multirow[t]{4}{*}{ Oral } & ASV687 & Flavobacteriales, Weeksellaceae, NA & 0.90 \\
\hline & & ASV843 & Myхососcales, $Р 3 O B-42, \mathrm{NA}$ & 0.92 \\
\hline & & ASV1161 & Pasteurellales, Pasteurellaceae, Phocoenobacter & 0.90 \\
\hline & & ASV1281 & Pseudomonadales, Moraxellaceae, NA & 0.98 \\
\hline Green & Oral \& Cloaca & ASV439 & Vibrionales, Vibrionaceae, NA & 0.92 \\
\hline \multirow[t]{3}{*}{ Kemp's ridley } & \multirow[t]{3}{*}{ Oral \& Cloaca } & ASV917 & Flavobacteriales, Flavobacteriaceae, NA & 0.95 \\
\hline & & ASV1104 & Campylobacterales, Arcobacteraceae, Arcobacter & 0.95 \\
\hline & & ASV1164 & Cardiobacteriales, Cardiobacteriaceae, Cardiobacterium & 0.92 \\
\hline
\end{tabular}



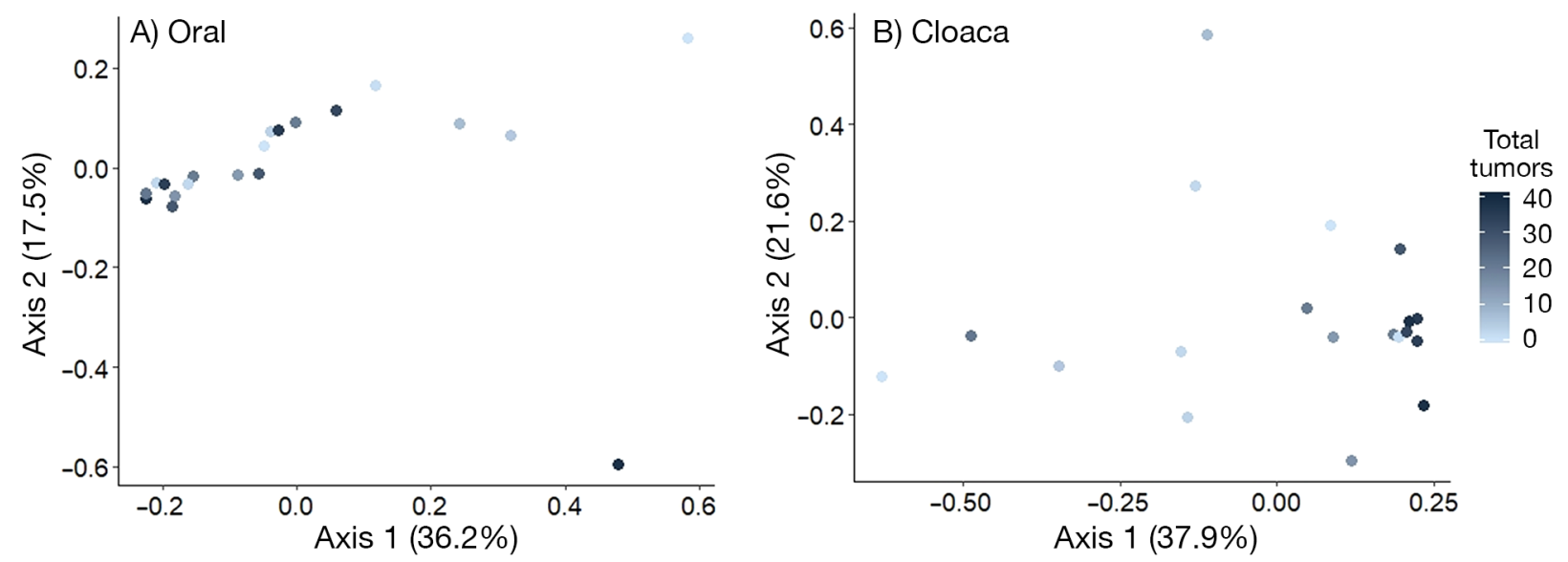

Fig. 6. Principal coordinate analysis (PCoA) plots of green turtle (A) oral (PERMANOVA, p = 0.175) and (B) cloaca (PERMANOVA, $p=0.008$ ) microbial communities based on Bray-Curtis dissimilarity and colored by total number of tumors
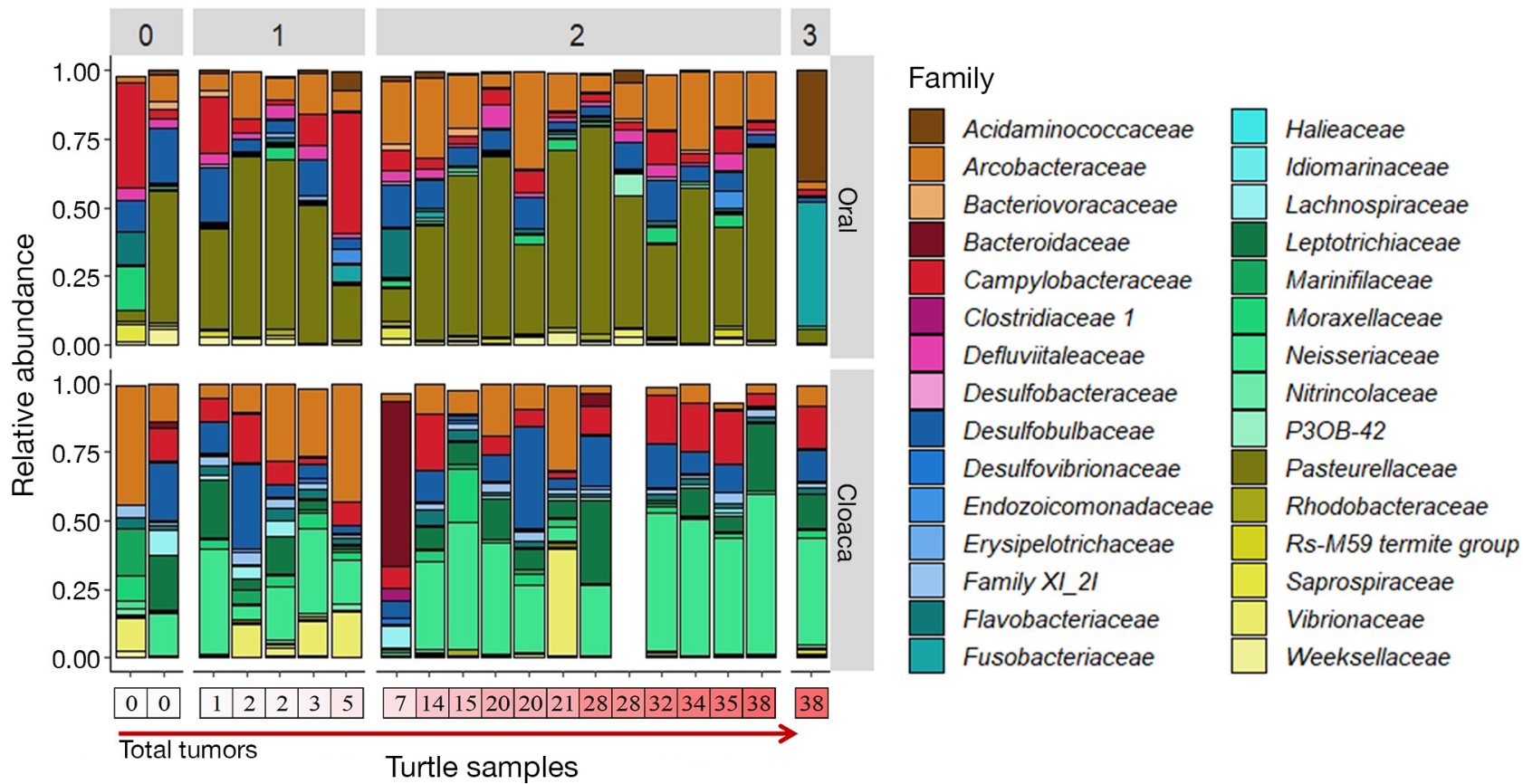

Fig. 7. Relative abundance of the top 30 families in samples of green turtles. Plots are separated by Balazs score and body site (oral or cloaca). Turtle samples are ordered by total number of tumors, with 0 tumors on the left to the most tumors (38) on the right

turtle cloaca samples but that was not present in green turtles. This family is also found in dolphin and whale respiratory vapor (Lima et al. 2012, Apprill et al. 2017) though its function in those environments is unknown. Cardiobacteriaceae may be responsible for human illnesses such as endocarditis and wound infections (Das et al. 1997), but pathogenicity in Kemp's ridley turtles is extremely unlikely due to its common presence and high abundance in seemingly healthy animals.
Several of these ASVs are part of the core microbiome (shared among $90 \%$ of samples) of the green turtle or Kemp's ridley turtle cloaca (Table 3). Additional members of the core microbiome include Lachnospiraceae, a family in the order Clostridiales, which was in $100 \%$ of the green turtle cloaca samples. This bacterial family consists of anaerobes with the ability to degrade polysaccharides, which is essential in herbivores such as marine iguanas and green turtles (Hong et al. 2011, Campos et al. 2018). 
Families in the order Clostridiales play a role in herbivore digestion by breaking down cellulose (Yuan et al. 2015), which is likely the reason multiple ASVs from this order constitute the cloacal microbiome of the herbivorous green turtle. Clostridiales has also been found in the cloacal and fecal microbiome of green turtles (Ahasan et al. 2017, Price et al. 2017, Campos et al. 2018, Bloodgood et al. 2020). We found Snodgrassella sp. (family Neisseriaceae) to be highly abundant in $100 \%$ of green turtle cloaca samples as well. Neisseriaceae is a common and diverse bacterial family inhabiting mucosal surfaces of humans and many other animals such as dogs, cats, dolphins, and iguanas (Liu et al. 2015). Identifying a core microbiome at the specific body site allows for comparison with data from other studies, by identifying microbiota that are likely healthy for the species rather than influenced by the environment. Investigating the functional core microbiome, including other sections of the GI tract, in the future would allow better insight into diet effects on these species.

There were no ASVs shared between the Kemp's ridley and green turtle cloaca samples, indicating their distinct microbiomes at this body site, which is likely due to their different diet requirements (carnivore vs. herbivore) and subsequent gut morphology (Ley et al. 2008a, Hong et al. 2011, Yuan et al. 2015, Campos et al. 2018). Presence of FP in green turtles may also be playing a role in the difference, although green turtles with no tumors were still different than the Kemp's ridley turtles. There were 4 ASVs from different families found in $90 \%$ of the oral samples from both turtle species. Two of these, Pasteurellaceae and Moraxellaceae, were also in high abundance across the oral samples. Pasteurellaceae may be more common than originally expected, as it has been found in human oral microbiome studies (Contreras et al. 2010), European bats (Mühldorfer et al. 2014), and the oral cavity of sea lions and walruses (Hansen et al. 2012). Specifically, Phocoenobacter sp., a genus within Pasteurellaceae that we found in the oral samples, was first described in a harbor porpoise uterus (Foster et al. 2000). Moraxellaceae is commonly found in the marine environment, but also includes species that colonize mucosal membranes or the skin of humans and animals (Teixeira \& Merquior 2014), including the oral cavity of dolphins (Bik et al. 2016) as well as the cloaca of green turtles (Price et al. 2017) and feces of loggerhead turtles (Arizza et al. 2019).

We found a high prevalence of FP in the green turtles we sampled, which may be due to the shallow/ inshore habitat, higher water temperatures in the summer months, biotoxin exposure, or unidentified water quality disturbances in this region (Jones et al. 2016, Page-Karjian \& Herbst 2017). There is weak clustering, based on Bray-Curtis dissimilarity, of cloacal microbial communities by total number of tumors in the green turtles (Fig. 6), but the small sample size makes this significance difficult to interpret. There was only 1 turtle with a Balazs score of 3 (the most severe) and this individual had a drastically different oral microbiome compared to other oral samples. In particular, Acidaminococcaceae were highly prevalent in this turtle due to $1 \mathrm{ASV}$, an Acidaminococcus sp. This bacterial genus is not well understood in animals, and although it increases in abundance in infants with chronic malnutrition (Gough et al. 2015), this turtle appeared to have good body condition. Fusobacterium sp. was also dominant in the oral cavity of the turtle with a Balazs score of 3, but it was found in low abundance in several other turtles with lower tumor scores. It has also been identified in loggerhead fecal samples, although not consistently or in much lower abundance compared to herbivores (Biagi et al. 2019), and we did not find it in the cloacal microbiome of any of the green turtles regardless of FP severity. Clustering by total tumors for cloaca samples may be driven by lower abundance of Vibrionaceae and Arcobacteraceae in the turtles with more tumors. Vibrio sp. were cultured in most turtles with increased severity of FP in Hawaii, USA (Work et al. 2003), but we found this genus to be in low abundance and not associated with FP severity. More turtles with severe cases of FP would need to be examined to determine whether the patterns observed here are, in fact, a direct result of FP. Location of the tumors on a turtle may also have a direct influence on the microbial communities at different body sites, either through physical contact with the tumors or by influencing exposure to transient bacteria from the local environment. The Balazs score, which is the most widely used scoring system in the field, only evaluates the number and size of the tumors, so future studies should examine a more clinically based scoring system (taking into account location on the turtle and morphology of the tumors) to further evaluate severity of the disease and effect on microbial communities (Page-Karjian et al. 2014, 2019, Page-Karjian \& Herbst 2017). Since we did find a significant relationship between FP and microbial community structure, despite our small sample size, additional investigations are essential to expand our identification of microbial correlations with disease and immune system function for this multifactorial disease in endangered sea turtles. 


\section{CONCLUSIONS}

We provide the first characterization of the oral and cloacal microbiome of 2 wild-caught sea turtle species, green and Kemp's ridley turtles, from the same environment, allowing us to identify differences in microbial community composition between species. We add microbiome data of green turtle cloaca samples to a growing field of studies and provide a first glimpse into the green turtle oral microbiome. We also provide valuable new information concerning the microbial composition of healthy Kemp's ridley turtles for both the oral cavity and cloaca from this endangered species. We identify a core microbiome for each species at each body site, allowing us to understand the potential importance of these microbes to the health of the turtle, including potential contributions to digestion based on diet. We also provide data on the correlation between the severity of FP in green turtles, and we identify the need for increased sample sizes and a higher resolution scoring system as important for further understanding the role of turtle microbiomes in health and disease. Understanding the microbiome from wild populations provides a foundational baseline for comparison that will allow for enhanced monitoring of sea turtle health in future studies, Massachusetts, USA.

Acknowledgements. We thank the New England Aquarium John H. Cunningham Award and an anonymous donor for funding the field portion of this study. We also thank the staff and volunteers of the Inwater Research Group. We thank Dr. Charles Innis for guidance throughout this study as well. Sequencing costs were supported with start-up funds to J.L.B. from Northeastern University, Massachusetts, USA.

\section{LITERATURE CITED}

Abdelrhman KFA, Bacci G, Mancusi C, Mengoni A, Serena F, Ugolini A (2016) A first insight into the gut microbiota of the sea turtle Caretta caretta. Front Microbiol 7:1060

Aguirre AA, Lutz PL (2004) Marine turtles as sentinels of ecosystem health: is fibropapillomatosis an indicator? EcoHealth 1:275-283

Ahasan MS, Waltzek TB, Huerlimann R, Ariel E (2017) Fecal bacterial communities of wild-captured and stranded green turtles (Chelonia mydas) on the Great Barrier Reef. FEMS Microbiol Ecol 93:fix139

Ahasan MS, Waltzek TB, Huerlimann R, Ariel E (2018) Comparative analysis of gut bacterial communities of green turtles (Chelonia mydas) pre-hospitalization and postrehabilitation by high-throughput sequencing of bacterial 16S rRNA gene. Microbiol Res 207:91-99

Ahasan MS, Waltzek TB, Owens L, Ariel E (2020) Characterisation and comparison of the mucosa-associated bacterial communities across gastrointestinal tract of stranded green turtles, Chelonia mydas. AIMS Microbiol 6:361-378
Amato KR (2013) Co-evolution in context: the importance of studying gut microbiomes in wild animals. Microbiome Sci Med 1:10-29

Apprill A, Robbins J, Eren AM, Pack AA and others (2014) Humpback whale populations share a core skin bacterial community: towards a health index for marine mammals? PLOS ONE 9:e90785

Apprill A, Miller CA, Moore MJ, Durban JW, Fearnbach H, Barrett-Lennard LG (2017) Extensive core microbiome in drone-captured whale blow supports a framework for health monitoring. mSystems 2:e00119-17

*Arizza V, Vecchioni L, Caracappa S, Sciurba G and others (2019) New insights into the gut microbiome in loggerhead sea turtles Caretta caretta stranded on the Mediterranean coast. PLOS ONE 14:e0220329

Arthur KE, Boyle MC, Limpus CJ (2008) Ontogenetic changes in diet and habitat use in green sea turtle (Chelonia mydas) life history. Mar Ecol Prog Ser 362: 303-311

Balazs GH (1991) Current status of fibropapillomatosis in the Hawaiian green turtle, Chelonia mydas. In: Balazs $\mathrm{GH}$, Pooley S (eds) Research plan for marine turtle fibropapilloma. US Department of Commerce, NOAA Technical Memorandum NMFS, NOAA-TM-NMFSSWFC-156, Honolulu, HI, p 47-57

Biagi E, D'Amico F, Soverini M, Angelini V and others (2019) Faecal bacterial communities from Mediterranean loggerhead sea turtles (Caretta caretta). Environ Microbiol Rep 11:361-371

*Bik EM, Costello EK, Switzer AD, Callahan BJ and others (2016) Marine mammals harbor unique microbiotas shaped by and yet distinct from the sea. Nat Commun 7: 10516

Bjorndal KA (1997) Foraging ecology and nutrition of sea turtles. In: Lutz PL, Musick JA (eds) The biology of sea turtles. CRC Press, Boca Raton, FL, p 199-231

* Bloodgood JCG, Hernandez SM, Isaiah A, Suchodolski JS and others (2020) The effect of diet on the gastrointestinal microbiome of juvenile rehabilitating green turtles (Chelonia mydas). PLOS ONE 15:e0227060

Bolten AB (2003) Variation in sea turtle life history patterns: neritic vs. oceanic developmental stages. In: Lutz PL, Musick JA, Wyneken J (eds) The biology of sea turtles, Vol II. CRC Press. Boca Raton, FL, p 243-257

Bordenstein SR, Theis KR (2015) Host biology in light of the microbiome: ten principles of holobionts and hologenomes. PLOS Biol 13:e1002226

Callahan BJ, McMurdie PJ, Rosen MJ, Han AW, Johnson AJ, Holmes SP (2016) DADA2: high-resolution sample inference from Illumina amplicon data. Nat Methods 13: 581-583

* Campos P, Guivernau M, Prenafeta-Boldu FX, Cardona L (2018) Fast acquisition of a polysaccharide fermenting gut microbiome by juvenile green turtles Chelonia mydas after settlement in coastal habitats. Microbiome 6:69

* Caporaso JG, Lauber CL, Walters WA, Berg-Lyons D and others (2012) Ultra-high-throughput microbial community analysis on the Illumina HiSeq and MiSeq platforms. ISME J 6:1621-1624

* Collado L, Figueras MJ (2011) Taxonomy, epidemiology, and clinical relevance of the genus Arcobacter. Clin Microbiol Rev 24:174-192

* Colston TJ, Jackson CR (2016) Microbiome evolution along divergent branches of the vertebrate tree of life: what is known and unknown. Mol Ecol 25:3776-3800 
Contreras M, Costello EK, Hidalgo G, Magris M, Knight R, Dominguez-Bello MG (2010) The bacterial microbiota in the oral mucosa of rural Amerindians. Microbiology 156: 3282-3287

Das M, Badley AD, Cockerill FR, Steckelberg JM, Wilson WR (1997) Infective endocarditis caused by HACEK microorganisms. Annu Rev Med 48:25-33

Eren AM, Vineis JH, Morrison HG, Sogin ML (2013) A filtering method to generate high quality short reads using Illumina paired-end technology. PLOS ONE 8:e66643

Foster G, Ross HM, Malnick H, Willems A, Hutson RA, Reid RJ, Collins MD (2000) Phocoenobacter uteri gen. nov., sp. nov., a new member of the family Pasteurellaceae Pohl (1979) 1981 isolated from a harbour porpoise (Phocoena phocoena). Int J Syst Evol Microbiol 50:135-139

Gough EK, Stephens DA, Moodie EEM, Prendergast AJ, Stoltzfus RJ, Humphrey JH, Manges AR (2015) Linear growth faltering in infants is associated with Acidaminococcus sp. and community-level changes in the gut microbiota. Microbiome 3:24

Hansen MJ, Bertelsen MF, Christensen H, Bojesen AM, Bisgaard M (2012) Otariodibacter oris gen. nov., sp. nov., a member of the family Pasteurellaceae isolated from the oral cavity of pinnipeds. Int J Syst Evol Microbiol 62: 2572-2578

Hong PY, Wheeler E, Cann IKO, Mackie RI (2011) Phylogenetic analysis of the fecal microbial community in herbivorous land and marine iguanas of the Galapagos Islands using 16S rRNA-based pyrosequencing. ISME J 5:1461-1470

Hoopes LA, Koutsos EA, Norton TM (2017) Nutrition. In: Manire CA, Norton TM, Stacy BA, Innis CJ, Harms CA (eds) Sea turtle health and rehabilitation. J. Ross Publishing, Plantation, FL, p 63-96

*Hyde ER, Navas-Molina JA, Song SJ, Kueneman JG and others (2016) The oral and skin microbiomes of captive Komodo dragons are significantly shared with their habitat. mSystems 1:e00046-16

Jones K, Ariel E, Burgess G, Read M (2016) A review of fibropapillomatosis in green turtles (Chelonia mydas). Vet J 212:48-57

Jooste PJ, Hugo CJ (1999) The taxonomy, ecology and cultivation of bacterial genera belonging to the family Flavobacteriaceae. Int J Food Microbiol 53:81-94

Keenan SW, Elsey RM (2015) The good, the bad, and the unknown: microbial symbioses of the American alligator. Integr Comp Biol 55:972-985

Keenan SW, Engel AS, Elsey RM (2013) The alligator gut microbiome and implications for archosaur symbioses. Sci Rep 3:2877

Lahti L, Shetty S (2017) Tools for microbiome analysis in R. Version 1.6.0. http://microbiome.github.com/microbiome

Ley RE, Hamady M, Lozupone C, Turnbaugh PJ and others (2008a) Evolution of mammals and their gut microbes. Science 320:1647-1651

Ley RE, Lozupone CA, Hamady M, Knight R, Gordon JI (2008b) Worlds within worlds: evolution of the vertebrate gut microbiota. Nat Rev Microbiol 6:776-788

Lima N, Rogers T, Acevedo-Whitehouse K, Brown MV (2012) Temporal stability and species specificity in bacteria associated with the bottlenose dolphins respiratory system. Environ Microbiol Rep 4:89-96

Liu G, Tang CM, Exley RM (2015) Non-pathogenic Neisseria: members of an abundant, multi-habitat, diverse genus. Microbiology 161:1297-1312
Love MI, Huber W, Anders S (2014) Moderated estimation of fold change and dispersion for RNA-seq data with DESeq2. Genome Biol 15:550

* McDermid KJ, Kittle RP III, Veillet A, Plouviez S, Muehlstein L, Balazs GH (2020) Identification of gastrointestinal microbiota in Hawaiian green turtles (Chelonia mydas). Evol Bioinform Online 16:1176934320914603

McFall-Ngai M, Hadfield MG, Bosch TCG, Carey HV and others (2013) Animals in a bacterial world, a new imperative for the life sciences. Proc Natl Acad Sci USA 110: 3229-3236

* McMurdie PJ, Holmes S (2013) phyloseq: an R package for reproducible interactive analysis and graphics of microbiome census data. PLOS ONE 8:e61217

*McNally KL, Mott CR, Guertin JR, Gorham JC, Innis CJ (2020) Venous blood gas and biochemical analysis of wild captured green turtles (Chelonia mydas) and Kemp's ridley turtles (Lepidochelys kempii) from the Gulf of Mexico. PLOS ONE 15:e0237596

* Mettel C, Kim Y, Shrestha PM, Liesack W (2010) Extraction of mRNA from soil. Appl Environ Microbiol 76:5995-6000

* Mühldorfer K, Speck S, Wibbelt G (2014) Proposal of Vespertiliibacter pulmonis gen. nov., sp. nov. and two genomospecies as new members of the family Pasteurellaceae isolated from European bats. Int J Syst Evol Microbiol 64:2424-2430

*Murali A, Bhargava A, Wright ES (2018) IDTAXA: a novel approach for accurate taxonomic classification of microbiome sequences. Microbiome 6:140

Page-Karjian A, Herbst LH (2017) Viruses. In: Manire CA, Norton TM, Stacy BA, Innis CJ, Harms CA (eds) Sea turtle health and rehabilitation. J. Ross Publishing, Plantation, FL, p 751-777

*Page-Karjian A, Norton TM, Krimer P, Groner M, Nelson SE, Gottdenker NL (2014) Factors influencing survivorship of rehabilitating green sea turtles (Chelonia mydas) with fibropapillomatosis. J Zoo Wildl Med 45:507-519

*Page-Karjian A, Perrault JR, Zirkelbach B, Pescatore J and others (2019) Tumor re-growth, case outcome, and tumor scoring systems in rehabilitated green turtles with fibropapillomatosis. Dis Aquat Org 137:101-108

Price JT, Paladino FV, Lamont MM, Witherington BE, Bates ST, Soule T (2017) Characterization of the juvenile green turtle (Chelonia mydas) microbiome throughout an ontogenetic shift from pelagic to neritic habitats. PLOS ONE 12:e0177642

R Core Team (2019) R: a language and environment for statistical computing. R Foundation for Statistical Computing, Vienna. www.R-project.org/

*Samuelson MM, Pulis EE, Ray C, Arias CR, Samuelson DR, Mattson EE, Solangi M (2020) Analysis of the fecal microbiome in Kemp's ridley sea turtles Lepidochelys kempii undergoing rehabilitation. Endang Species Res 43:121-131

* Scheelings TF, Moore RJ, Van TTH, Klaassen M, Reina RD (2020a) Microbial symbiosis and coevolution of an entire clade of ancient vertebrates: the gut microbiota of sea turtles and its relationship to their phylogenetic history. Anim Microbiome 2:17

K Scheelings TF, Moore RJ, Van TTH, Klaassen M, Reina RD (2020b) No correlation between microbiota composition and blood parameters in nesting flatback turtles (Natator depressus). Sci Rep 10:8333

* Scheelings TF, Moore RJ, Van TTH, Klaassen M, Reina RD (2020c) The gut bacterial microbiota of sea turtles differs 
between geographically distinct populations. Endang Species Res 42:95-108

Seminoff JA (Southwest Fisheries Science Center, US) (2004) Chelonia mydas. The IUCN Red List of Threatened Species 2004:e.T4615A11037468 (accessed 20 July 2019)

Shade A, Handelsman J (2012) Beyond the Venn diagram: the hunt for a core microbiome. Environ Microbiol 14:4-12

Teixeira LM, Merquior VLC (2014) The family Moraxellaceae. In: Rosenberg E, DeLong EF, Lory S, Stackebrandt E, Thompson F (eds) The prokaryotes. Springer, Berlin, p 443-476

Turnbaugh PJ, Ley RE, Hamady M, Fraser-Liggett CM, Knight R, Gordon JI (2007) The Human Microbiome Project. Nature 449:804-810

Editorial responsibility: Richard Reina,

Clayton, Victoria, Australia

Reviewed by: 3 anonymous referees
Wibbels T, Bevan E (2019) Lepidochelys kempii. The IUCN Red List of Threatened Species 2019:e.T11533A142050590 (accessed 24 September 2019)

*Work TM, Balazs GH, Wolcott M, Morris R (2003) Bacteraemia in free-ranging Hawaiian green turtles Chelonia mydas with fibropapillomatosis. Dis Aquat Org 53: $41-46$

Wyneken J, Mader D, Weber S, Merigo C (2006) Medical care of sea turtles. In: Mader, DM (ed) Reptile medicine and surgery, 2nd edn. Elsevier, St. Louis, MO, p 972-1007

* Yuan ML, Dean SH, Longo AV, Rothermel BB, Tuberville TD, Zamudio KR (2015) Kinship, inbreeding and finescale spatial structure influence gut microbiota in a hindgut-fermenting tortoise. Mol Ecol 24:2521-2536

Submitted: October 13, 2020

Accepted: January 28, 2021

Proofs received from author(s): May 2, 2021 